\title{
Antibiotic treatment is associated with reduced risk of a subsequent exacerbation in obstructive lung disease: an historical population based cohort study
}

\author{
B M Roede, ${ }^{1,2}$ P Bresser, ${ }^{3}$ P J E Bindels, ${ }^{2}$ A Kok, ${ }^{4}$ M Prins, ${ }^{1,4} \mathrm{G}$ ter Riet, ${ }^{2,5}$ R B Geskus, \\ R M C Herings, ${ }^{7} \mathrm{~J}$ M Prins $^{1}$
}

\begin{abstract}
See Editorial p 940
${ }^{1}$ Department of Internal

Medicine, Division of Infectious Diseases, Tropical Medicine and AIDS, and Centre for Infection and Immunity Amsterdam (CINIMA), Amsterdam, The Netherlands; ${ }^{2}$ Department of General Practice, Academic Medical Centre-University of Amsterdam, Amsterdam, The Netherlands; ${ }^{3}$ Department of Pulmonology, Academic Medical Centre-University of Amsterdam, Amsterdam, The Netherlands;

${ }^{4}$ Municipal Health Service, Cluster Infectious Diseases, Amsterdam, The Netherlands;

${ }^{5}$ Horten Centre, University of Zurich, Zurich, Switzerland;

${ }^{6}$ Department of Clinical Epidemiology and Biostatistics, Academic Medical CentreUniversity of Amsterdam, Amsterdam, The Netherlands; ${ }^{7}$ PHARMO Institute, Utrecht, The Netherlands
\end{abstract}

Correspondence to: B M Roede, Academic Medical Centre-University of Amsterdam, Department of Internal Medicine, Division of Infectious Diseases, Tropical Medicine and AIDS, and Centre for Infection and Immunity Amsterdam (CINIMA), F4-217, Meibergdreef 9, 1105 AZ Amsterdam, The Netherlands; i.roede@ amc uva nl

Received 2 January 2008 Accepted 8 June 2008 Published Online First

5 August 2008

\begin{abstract}
Objectives: The risk of a subsequent exacerbation after treatment of an exacerbation with oral corticosteroids without (OS) or with (OSA) antibiotics was evaluated in a historical population based cohort study comprising patients using maintenance medication for obstructive lung disease.
\end{abstract}

Methods: The Pharmo database includes drug dispensing records of more than 2 million subjects in The Netherlands. Eligible were patients $\geqslant 50$ years who in 2003 were dispensed $\geqslant 2$ prescriptions of daily used inhaled $\beta_{2}$ agonists, anticholinergics and/or corticosteroids, and experienced at least one exacerbation before 1 January 2006. Exacerbation was defined as a prescription of OS or OSA. The times to the second and third exacerbations were compared using Kaplan-Meier survival analysis. Independent determinants of new exacerbations were identified using multivariable Cox recurrent event survival analysis.

Results: Of 49599 patients using maintenance medication, 18928 had at least one exacerbation; in 52\%, antibiotics had been added. The OS and OSA groups were comparable for potential confounding factors. Median time to the second exacerbation was 321 days in the OS group and 418 days in the OSA group $(p<0.001)$; and between the second and third exacerbation 127 vs 240 days $(p<0.001)$. The protective effect of OSA was most pronounced during the first 3 months following treatment (hazard ratio (HR) 0.62; $99 \% \mathrm{Cl} 0.60$ to 0.65 ). In the OSA group, mortality during follow-up was lower (HR 0.82; $99 \% \mathrm{Cl} 0.66$ to 0.98 ).

Conclusion: Treatment with antibiotics in addition to oral corticosteroids was associated with a longer time to the next exacerbation, and a decreased risk of developing a new exacerbation.

The mainstay of treatment of exacerbations of chronic obstructive pulmonary disease (COPD) consists of oral corticosteroids and antibiotics. The use of corticosteroids in this situation is well accepted, but the role of antibiotics in exacerbations of COPD is still debated. Patients with severe exacerbations, characterised by severe symptoms, and/or patients with a low baseline expiratory flow rate, are considered to benefit from antibiotic treatment. ${ }^{1-3}$ A recent Cochrane review supports the use of antibiotics for short term benefits in patients with an exacerbation with increased cough and sputum purulence who are moderately or severely ill. ${ }^{4}$ Studies conducted in general practice showed no advantage of antimicrobial treatment. ${ }^{5-7}$ Given the inconclusive results from the literature, guidelines differ in their recommendations. ${ }^{8-12}$ The main concern with antibiotic use is a rise in antimicrobial resistance, which correlates well with the overall antibiotic use in the community. ${ }^{13}$

The Dutch Pharmo database contains extensive data on pharmacy dispensing records from community pharmacies and hospital discharge records of more than 2 million residents of The Netherlands. ${ }^{14}$ This gave us the unique opportunity to evaluate the long term effects of treatment of exacerbations in a large patient group using maintenance medication for obstructive lung disease. In particular, we compared the risk of a subsequent exacerbation after treatment with oral corticosteroids without or with antibiotics.

\section{METHODS}

\section{Data sources}

Data for this study were obtained from the Pharmo database. ${ }^{14}$ This population based database includes pharmacy dispensing records from community pharmacies and hospital discharge records of more than 2 million residents of 50 regions scattered over The Netherlands. Data are representative for The Netherlands. Both prescriptions from general practitioners and outpatients are registered. For all residents, the drug dispensing histories are linked to the hospital discharge records of the same patient, using a probabilistic algorithm, based on characteristics such as date of birth, gender and a code for the general practitioner. ${ }^{15}$ The computerised drug dispensing histories contain data concerning the dispensed drug and dispensing date. Drugs are coded according to the Anatomical Therapeutic Chemical (ATC) classification system. ${ }^{16}$ The hospital records include information concerning the primary and secondary diagnoses, procedures and dates of hospital admission and discharge. All diagnoses are coded according to the International Classification of Diseases, 9th revision, Clinical Modification (ICD-9-CM).

For this study, ethics approval was not relevant because data were anonymised before entering the Pharmo database.

\section{Patient selection}

We included patients who in 2003 were dispensed at least two prescriptions of daily used respiratory drugs with ATC-code R03 (ie, inhaled $\beta_{2}$ agonists, inhaled anticholinergics, inhaled corticosteroids, oral theophylline or a combination of these 
Table 1 Characteristics of patients according to treatment of first exacerbation

\begin{tabular}{|c|c|c|c|c|}
\hline & $\begin{array}{l}\text { Total } \\
(\mathrm{n}=18928)\end{array}$ & $\begin{array}{l}\text { Oral } \\
\text { corticosteroids } \\
(\mathrm{n}=9074)\end{array}$ & $\begin{array}{l}\text { Oral corticosteroids } \\
\text { and antibiotics } \\
(\mathrm{n}=9854)\end{array}$ & p Value : \\
\hline \multicolumn{5}{|l|}{ Gender } \\
\hline Male & $9395(50)$ & $4536(50)$ & $4859(49)$ & 0.35 \\
\hline Female & $9533(50)$ & $4538(50)$ & $4995(51)$ & \\
\hline Age (years) & $70(61-77)$ & $70(61-77)$ & $70(61-77)$ & 0.60 \\
\hline No of respiratory dispensings in 2003 & $8(4-13)$ & $8(4-13) \S$ & $8(4-13) \S$ & $<0.01$ \\
\hline ICS (maintenance medication) & $16771(89)$ & $7883(87)$ & $8888(90)$ & $<0.01$ \\
\hline \multicolumn{5}{|l|}{ Co-medication } \\
\hline Cardiovascular & 12995 (69) & $6245(69)$ & $6750(68)$ & 0.63 \\
\hline Diabetes & $2682(14)$ & $1264(14)$ & $1418(14)$ & 0.36 \\
\hline \multicolumn{5}{|l|}{ Hospitalisation* for } \\
\hline $\mathrm{COPD} \dagger$ & $1925(10)$ & $994(11)$ & 931 (9) & $<0.01$ \\
\hline Pneumonia & $651(3)$ & $325(4)$ & $326(3)$ & 0.30 \\
\hline \multicolumn{5}{|l|}{ Follow-up characteristics } \\
\hline Follow-up time (days) & 754 (437-974) & 786 (472-988) & 739 (404-957) & \\
\hline Number of exacerbations & $2(1-4)$ & $2(1-4)$ & $2(1-3)$ & \\
\hline
\end{tabular}

Data are $\mathrm{n}(\%)$ or median (interquartile range).

${ }^{*}$ Once or more in previous 2 years. †Diagnosis: chronic bronchitis, emphysema or COPD. $\$ \chi^{2}$ or Mann-Whitney test, where appropriate. §The oral corticosteroids and antibiotics group had a significantly higher number of respiratory dispensings, but the 25th, 50th and 75th percentiles for both treatment groups were similar.

COPD, chronic obstructive pulmonary disease; ICS, inhaled corticosteroids.

agents). We included patients aged 50 years or older in order to exclude patients with uncomplicated asthma.

Furthermore, patients using leukotriene receptor antagonists (LTRAs) (montelukast, ATC-code R03DC03) or cromoglycates (R03BC) and patients who had been hospitalised with a diagnosis of asthma (ICD-9-CM) in the previous 2 years or during follow-up were excluded.

The cohort entry date was the date of first dispensing of any R03 drug in 2003. Patients were followed until 31 December 2005. If patients died before that date, they were censored at the date of death. Apart from age, sex and respiratory drugs, data on co-medication for cardiovascular disease and diabetes mellitus, as well as previous hospitalisations for COPD and pneumonia were collected.

\section{Definition of exacerbation}

To address the study question, we identified those patients who experienced one or more exacerbations. Our assumption is that, in these patients who are on respiratory drugs, a short course of oral corticosteroids is almost exclusively prescribed in the case of an exacerbation. Therefore, we defined an exacerbation as a documented dispensing of a short course of oral corticosteroids, with or without antibiotics. We documented the date of dispensing of oral corticosteroids (ATC code: H02AB06/ H02AB07), with or without antibiotics. We scored the antibiotics doxycyclin (ATC-code: J01AA02), amoxicillin (J01CA04), amoxicillin-clavulanate (J01CR02), azithromycin (J01FA10), clarithromycin (J01FA09), ciprofloxacin (J01MA02), moxifloxacin (J01MA14), levofloxacin (J01MA12) and erythromycin (J01FA01) because in The Netherlands these cover almost all antibiotics dispensed for exacerbations of COPD. ${ }^{17} \mathrm{We}$ did not include episodes treated only with antibiotics, because information on the coinciding diagnosis was unavailable.

As symptoms, increased at the onset of exacerbation, are usually substantially improved after 3 weeks, ${ }^{18}$ we presumed that a second prescription for steroids within 3 weeks suggested an exacerbation not responding well to the initial therapy. To avoid counting these prescriptions as a "next exacerbation", we introduced a minimum interval between steroid courses of 3 weeks. If the time between two dispensings exceeded 3 weeks, we considered the second episode as a new exacerbation. As a consequence, until 3 weeks after the first course was dispensed, patients were considered not to be at risk for a new exacerbation. Patients dispensed oral corticosteroids or antibiotics for more than 21 days at regular intervals, for a period of 3 months or longer, were excluded from the analysis because they are likely to be patients on maintenance treatment with oral corticosteroids or antibiotics, respectively.

\section{Statistical analysis}

We assessed the first exacerbation after cohort entry (first course of oral corticosteroids) and calculated the time to the second exacerbation (second course of oral corticosteroids). Similarly, we calculated the time between the second and third exacerbation. We compared these time periods between patients treated with oral corticosteroids only, and those treated with oral corticosteroids combined with antibiotics using Kaplan-Meier survival analysis. Patients were censored for exacerbation free survival. The effect of the variable of primary interest, oral corticosteroid (coded as 0 ) or oral corticosteroid with antibiotic (coded as 1), was analysed in a Cox proportional hazards model. All exacerbations from each patient were used, and time was set back to zero after each exacerbation (gap time unrestricted model). ${ }^{19}$ Hence each exacerbation was treated as a separate record and time since last exacerbation as the principal time scale. A correction for recurrent exacerbation events from the same individual was made by including a frailty term in the model ${ }^{20}$ The Schoenfeld residuals, as obtained from the model of time to first exacerbation, suggested the difference in treatment effect be highly non-proportional. Therefore, we allowed the difference in treatment effect to change at 3 months, 6 months and 1 year. The data were coded so that hazard ratios below unity indicated a preventive effect of adding an antibiotic to the oral corticosteroids. Potential confounding by the following factors was controlled for: sex, age, number of dispensings of 


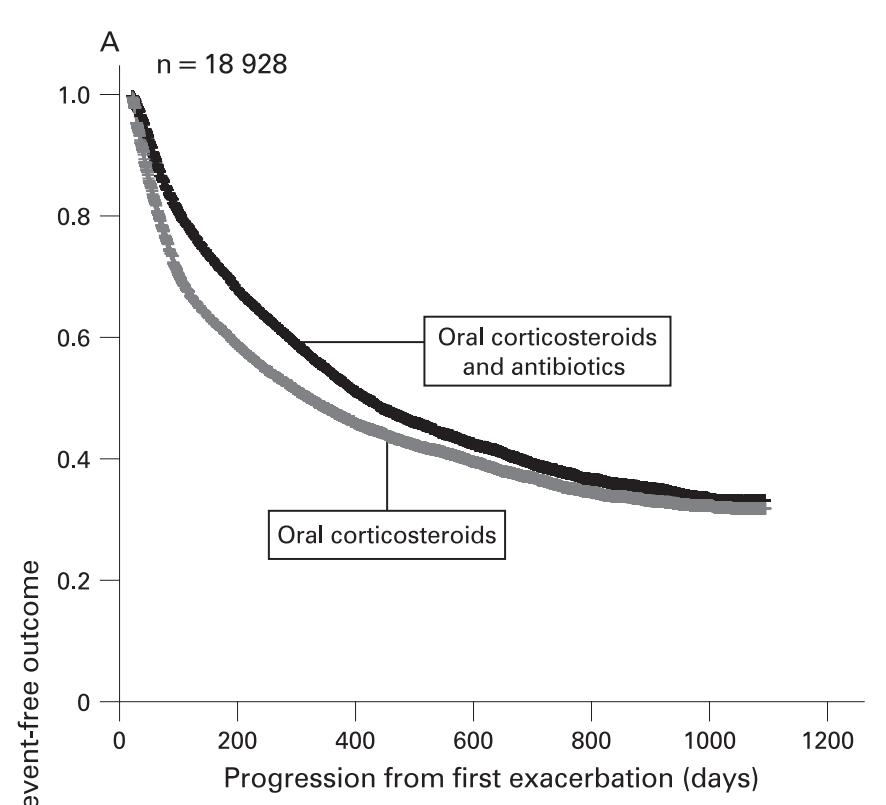

B

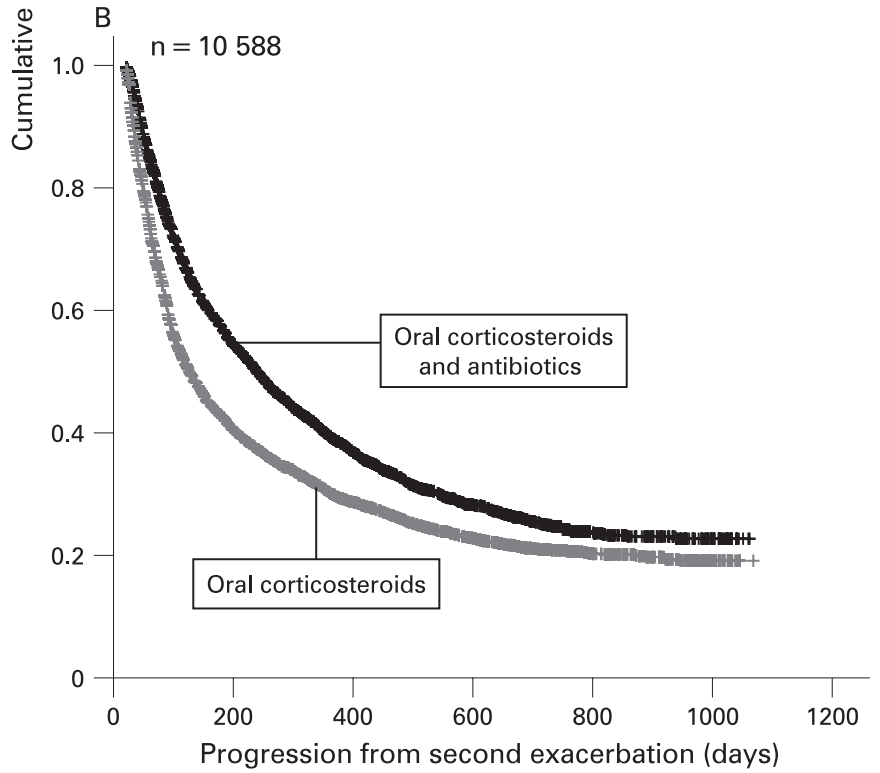

Figure 1 Kaplan-Meier estimates of the cumulative incidence of developing a second $(A)$ or third $(B)$ exacerbation stratified according to treatment type.

respiratory drugs, including inhaled corticosteroids, co-medication for cardiovascular disease (yes/no) or for diabetes (yes/no), and previous hospitalisation for COPD and pneumonia (yes/ no). ${ }^{21}$ Dispensing of antibiotics unrelated to exacerbations was also treated as a time dependent covariate, and assumed to be of influence for a period of 3 months. This means that 3 months after dispensing this antibiotic, the variable was again coded as no antibiotic. All cause mortality of both treatment groups was analysed using Kaplan-Meier survival analysis and Cox regression analysis. We calculated 99\% confidence interval (CI). Analyses were performed using Stata software, V.9.2 (StataCorp, College Station, Texas, USA), R-2.6.0 (R Development Core Team (2007). R: A language and environment for statistical computing. $R$ Foundation for Statistical Computing, Vienna, Austria. ISBN 3-900051-07-0, URL http://www.R-project.org) and SPSS V.14.0.2 software (SPSS, Chicago, Illinois, USA).

\section{RESULTS}

\section{Patients}

From the Pharmo database we identified 52753 patients, 50 years of age and older, fulfilling the prespecified criteria on respiratory drug use. In total, 3114 patients who had been hospitalised with a diagnosis of asthma or used LTRAs or cromoglycates were excluded, and 40 patients were excluded because of administrative errors, leaving 49599 patients. Of these patients, $19882(40 \%)$ had suffered at least one exacerbation as previously defined. A total of 715 patients were excluded from further analysis because they were likely to be on maintenance treatment with oral corticosteroids $(n=349)$, or with antibiotics $(n=366) ; 239$ patients were not at risk of a next exacerbation as they had only one exacerbation within the 3 weeks before the end of the follow-up, leaving 18928 patients for further analysis.

We found that 1053 patients (6\%) died during the follow-up period. For 894 patients the cause of death was unknown; 159 patients died during hospitalisation for COPD. This was counted as an event, and not right censored. After the first exacerbation, 2341 hospital admissions for COPD occurred; 1636 within 1 month of a documented exacerbation and 546 independently from a registered exacerbation. These 546 hospital admissions were considered as an event; instead of "time to the next exacerbation", time to hospital admission was counted. As information on inhospital treatment was not available, they were not analysed further.

The median follow-up time after the first exacerbation was 754 days (interquartile range (IOR) 437-974). In total, 18928 patients were followed for 36104 person years. General practitioners prescribed $72 \%$ of all antibiotics, oral corticosteroids and respiratory maintenance medication, $24 \%$ came from pulmonologists and $5 \%$ from other or unknown prescribers. Antibiotics used in the treatment of first exacerbations were doxycyclin ( $\mathrm{n}=4011,41 \%$ ), penicillins (amoxicillin-clavulanate and amoxicillin, $\mathrm{n}=3597,37 \%$ ), macrolides (azithromycin and clarithromycin, $\mathrm{n}=1916,20 \%$ ) and fluoroquinolones (ciprofloxacin, moxifloxacin and levofloxacin, $n=260,3 \%$ ). Fiftythree cases could not be assigned to a treatment group.

\section{Kaplan-Meier estimates of the cumulative incidence of developing a second or third exacerbation stratified according to treatment type (oral corticosteroids with or without antibiotics)} Time to second exacerbation

Of 18928 patients having had at least one exacerbation, the first exacerbation after cohort entry was treated with oral corticosteroids only in 48\% (9074 patients) and with oral corticosteroids and antibiotics in 52\% (9854 patients). These two groups of patients were similar with respect to age, gender, use of co-medication for diabetes and cardiovascular disease, and the number of previous hospitalisations for pneumonia. However, more patients in the oral corticosteroids with antibiotics group were dispensed prescriptions of respiratory maintenance drugs and inhaled corticosteroids over time $(p<0.01)$. On the other hand, this group had suffered fewer hospital admissions for COPD in the previous 2 years compared with patients in the oral corticosteroids only group $(p<0.01)$ (table 1).

The time to the second exacerbation was much shorter in the oral corticosteroids only group than in the oral corticosteroids with antibiotics group (fig 1A). Median time between the first and second exacerbation in the oral corticosteroids only group was 321 days (99\% CI 297 to 345) compared with 418 (99\% CI 393 to 443 ) days in the oral corticosteroids with antibiotics 
group. Six months after the first exacerbation, $60 \%$ in the oral corticosteroids only group had suffered no new exacerbation compared with $70 \%$ in the oral corticosteroids with antibiotics group. Twelve months after the first exacerbation this was $48 \%$ and $54 \%$, respectively. There were no differences between antibiotic treatment groups with respect to time to the second exacerbation. Median time to the second exacerbation was 414 (99\% CI 377 to 451) days for doxycyclin, 415 (371 to 458) days for penicillins, 431 (376 to 486) days for macrolides and 329 (189 to 469$)$ days for fluoroquinolones $(p=0.14)$.

During follow-up, $472 / 9854$ (4.8\%) patients died in the oral corticosteroid and antibiotic group versus 581/9074 (6.4\%) in the corticosteroid only group $(p<0.01)$ (fig 2$)$. In a univariate Cox regression model, the hazard ratio (HR) of mortality after treatment with oral corticosteroids and antibiotics compared with corticosteroids only was 0.78 (99\% CI 0.62 to 0.94$)$. In a multivariable Cox model, adjusting for potential confounders (sex, age, number of dispensings of respiratory drugs, including inhaled corticosteroids, co-medication for cardiovascular disease or for diabetes, previous hospitalisation for COPD and pneumonia, and exposure to antibiotics unrelated to exacerbation), the HR was 0.82 (99\% CI 0.66 to 0.98 ).

\section{Time to third exacerbation}

Of the 18928 patients with a first exacerbation, 10588 had suffered a second exacerbation. Of these exacerbations, 5420 (51\%) were treated with oral corticosteroids and 5168 (49\%) with oral corticosteroids and antibiotics. After the second exacerbation the difference between the two treatment groups with respect to the time to the next (third) exacerbation was even more pronounced than after the first exacerbation (fig 1B). The median time between the second and third exacerbation was 127 (99\% CI 117 to 137) days in the oral corticosteroids only group and 240 (99\% CI 222 to 258) days in the oral corticosteroids with antibiotics group. Six months after the second exacerbation, $42 \%$ of patients treated with oral corticosteroids had not had a third exacerbation compared with $57 \%$ of patients treated with oral corticosteroids and antibiotics; after 1 year this was $30 \%$ and $39 \%$, respectively.

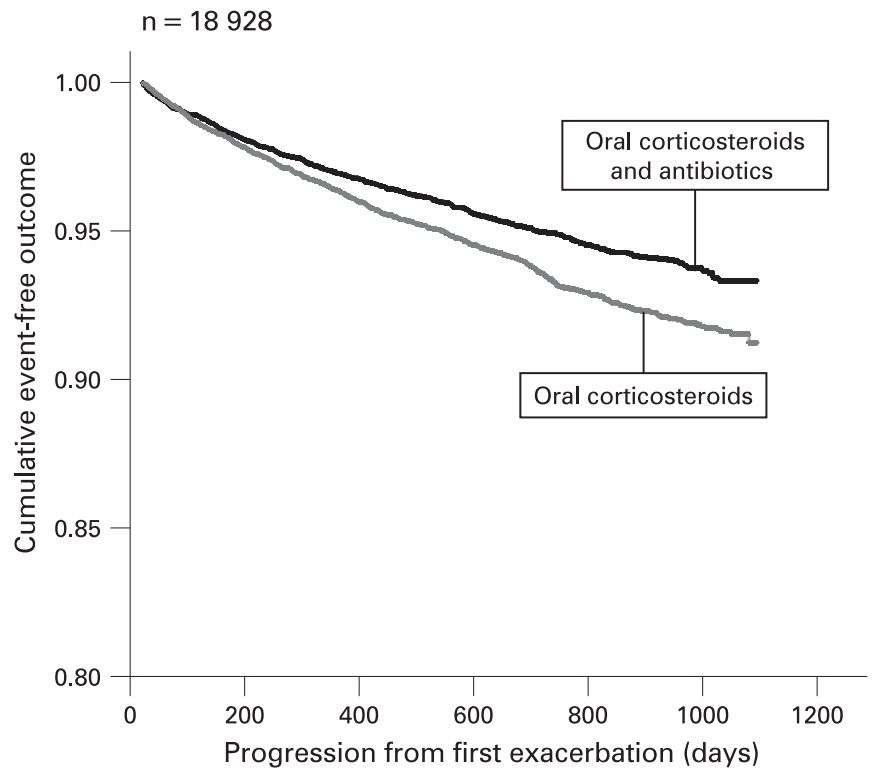

Figure 2 Kaplan-Meier estimates of the cumulative survival stratified according to treatment type.

\section{Cox recurrent event survival analysis of developing new exacerbations: effect of treatment type}

In a univariate Cox regression model, the $\mathrm{HR}$ of a new exacerbation after treatment with oral corticosteroids and antibiotics compared with corticosteroids only was $0.63(99 \%$ CI 0.61 to 0.66 ) in the first 3 months following treatment. In a multivariable Cox model adjusting for potential confounders, the HR of a new exacerbation after treatment with corticosteroids with antibiotics was 0.62 (99\% CI 0.60 to 0.65$)$ in the first 3 months following treatment, but the effect difference decreased in subsequent time periods (table 2). Exposure to antibiotics unrelated to exacerbations decreased the risk of a new exacerbation (HR $0.82 \quad(99 \%$ CI 0.78 to 0.87$)$ ). Hospitalisation for COPD in the previous 2 years increased the risk (HR 1.45 (99\% CI 1.35 to 1.57)). The variables "age" and "number of respiratory drugs dispensings in 2003" were included in the Cox model, but were not fitted linearly, and therefore HR are not presented. The risk of a new exacerbation increased with age until 80 years and with a higher number of respiratory drugs dispensings in 2003 up to 50 dispensings, but decreased after these values.

\section{Checking the assumption that a short course of oral corticosteroids in patients who are on respiratory drugs is dispensed in case of an exacerbation of COPD}

We defined an exacerbation as a course of oral corticosteroids (with or without antibiotics), assuming that (in The Netherlands) a course of oral corticosteroids in this population of patients on maintenance respiratory drugs is almost exclusively prescribed because of an exacerbation of COPD. We checked this assumption by investigating data based on the Second Dutch National Survey of General Practice (DNSGP-2), carried out by The Netherlands Institute for Health Services Research (NIVEL). This registration database gives a representative impression of morbidity and prescribing habits in Dutch general practice..$^{22}$ From 1 January 2002 to 31 December 2002, from 1037 patients with COPD defined according to the International Classification of Primary Care (ICPC) code $\mathrm{R} 95,{ }^{23}$ we analysed 1355 prescriptions of oral corticosteroids. A total of 1254 prescriptions (92\%) were COPD related. Forty prescriptions (3\%; upper limit of the 95\% CI 4.0) were prescribed for "other musculoskeletal/connective disorders" (ICPC-code L99). In addition, 61 prescriptions (4\%; upper limit of the $95 \%$ CI 5.7) were prescribed for other indications. Extrapolating these findings to the present study, we believe our definition of exacerbation was appropriate, and this small percentage of misclassified patients would cause bias towards the null.

\section{DISCUSSION}

In this historical follow-up study among 18928 patients with an exacerbation of obstructive lung disease, and focusing on relapse and not on short term recovery, we showed that treatment with oral corticosteroids and antibiotics compared with treatment with oral corticosteroids alone was associated with a longer time to the next exacerbation, and a decreased risk of developing a new exacerbation. Exposure to antibiotics between exacerbations was also associated with a lower risk of a subsequent exacerbation. In addition, in the group also treated with antibiotics, mortality during follow-up was significantly lower. As in the majority of cases the cause of death was unknown, we are cautious to claim a survival benefit, but this 
Table 2 Hazard ratios of determinants of developing a next exacerbation after oral corticosteroids with antibiotics - compared with oral corticosteroids only — treatment in a multivariable Cox model

\begin{tabular}{llll}
\hline & $\begin{array}{l}\text { HR of new } \\
\text { exacerbation }\end{array}$ & & \multicolumn{2}{c}{$\mathbf{9 9 \%}$ Cl for hazard ratio } \\
\cline { 4 - 4 } & & Lower & Upper \\
\hline Antibiotics added to treatment with oral corticosteroids & & & \\
0-3 months following treatment & 0.62 & 0.60 & 0.65 \\
3-6 months following treatment & 0.68 & 0.65 & 0.73 \\
6-12 months following treatment & 1.03 & 0.96 & 1.12 \\
$>12$ months following treatment & 1.31 & 1.18 & 1.45 \\
Exposure to antibiotics after previous exacerbation & 0.82 & 0.78 & 0.87 \\
Female sex & 0.95 & 0.91 & 1.00 \\
Inhaled corticosteroids as maintenance medication & 0.91 & 0.84 & 0.98 \\
Co-medication cardiovascular & 1.16 & 1.10 & 1.23 \\
Co-medication for diabetes & 1.05 & 0.98 & 1.12 \\
Hospitalisation* for COPD $\dagger$ & 1.45 & 1.35 & 1.57 \\
Hospitalisation for pneumonia & 1.19 & 1.05 & 1.34
\end{tabular}

The variables "age" and "number of respiratory drugs dispensings in 2003" were included in the Cox model, but were not fitted linearly, and therefore HR are not presented.

${ }^{*}$ Once or more in previous 2 years. †Diagnosis: chronic bronchitis, emphysema or COPD.

COPD, chronic obstructive pulmonary disease; HR, hazard ratio.

finding is important and certainly warrants confirmation in a prospective study.

Deriving the data from a pharmacy database enabled us to measure actually dispensed medication in a very large number of patients treated with maintenance treatment for obstructive lung disease. Prescriptions both from general practitioners and pulmonologists were documented.

A limitation of observational studies might be the presence of treatment selection bias as a result of unknown potentially prognostic important differences among patients. ${ }^{24}$ In our study, clinical information on patients was not available. However, patients treated with antibiotics and oral steroids are likely to have more severe exacerbations compared with patients treated with oral steroids only. Therefore, we suspect that any treatment selection bias, if present, would cause bias towards the null, thus underestimating the effect of adding antibiotics.

We found that the effect of treatment was strongest in the first 3 months following treatment and then gradually decreased, and even reversed after 1 year. Apparently, the protective effect of antibiotics wanes over time, which is to be expected.

Patients could not be selected based on a diagnosis of COPD or chronic bronchitis. Therefore, we selected patients who used maintenance respiratory drugs used in the treatment of obstructive lung disease. We included patients aged 50 years or older, in order to exclude patients with uncomplicated asthma. Furthermore, we excluded patients who had been hospitalised with a diagnosis of asthma and those using LTRAs or cromoglycates. Nevertheless, a small proportion of patients with asthma may still have been included in this study.

Recent studies stress the benefits from antibiotic treatment in exacerbations of $\mathrm{COPD},{ }^{25}$ and a recent Cochrane review reports reduction of mortality and treatment failure, although in community based studies no differences were found between antibiotics and placebo. ${ }^{4}$ Most studies so far have been conducted in clinical settings, frequently in hospitalised patients, with merely severe exacerbations. For patients treated in general practice, studies showed no advantage of antimicrobial treatment on short term outcome..$^{5-7}$ In the population we studied, general practitioners were responsible for $72 \%$ of all dispensings. In general, these patients with COPD can be classified as GOLD 2 (moderate COPD) ${ }^{26}$ Therefore, in patients with less severe exacerbations, antibiotic treatment added to treatment with oral corticosteroids also seems advantageous. The differences we found with respect to time from the first to the second exacerbation and from the second to the third exacerbation suggest that in patients with frequent exacerbations the benefits of antibiotic treatment added to treatment with oral corticosteroids may be greater.

Most randomised trials have follow-up durations of no more than 6 weeks. ${ }^{27}$ Benefits from antibiotic treatment may become more apparent in the long term follow-up compared with short term evaluations, possibly because of antibiotic treatment decreasing bacterial load. The finding that exposure to antibiotics for any indication also decreased the risk of a next exacerbation may suggest that some patients with COPD carrying bacteria in a stable state could benefit from antibiotics.

There is sufficient evidence for the contributory role of bacteria in exacerbations. During bacterial exacerbations, bacteria are present in the lower airways, associated with airway inflammation, and in sufficient concentrations $(>1000 \mathrm{cfu} / \mathrm{ml})$ to cause invasive infections. ${ }^{28}$ Inflammatory changes are also related to recurrent exacerbations. ${ }^{29}$ Immune responses to bacteria play an important role, and non-typeable Haemophilus influenzae in particular is known for its role in the process of colonisation and infection. ${ }^{30-33}$ Isolation of new strains of bacterial pathogens increases the risk of an exacerbation. ${ }^{34}$ Increased sputum purulence is the main sign of a new or increased significant bacterial stimulus. ${ }^{25} 283536$ In addition, patients with severe exacerbations are known to benefit most from antibiotic treatment. ${ }^{1-4}$

We showed that treatment with oral corticosteroids and antibiotics compared with treatment with oral corticosteroids alone was associated with a longer time to the next exacerbation, and a decreased risk of developing a new exacerbation. On the other hand, treating all exacerbations with antibiotics will significantly increase overall antibiotic consumption, which might fuel the increasing rates of resistance among respiratory pathogens. ${ }^{37-41}$

Therefore, future prospective studies should explore for which exacerbations with respect to patient profiles and clinical symptoms in particular antibiotics are indicated.

Acknowledgements: Author contributions: BMR had full access to all of the data in the study and takes responsibility for the integrity of the data and the accuracy of the 
data analysis. Study concept and design: JP, PB and PJEB. Acquisition of data: RH. Analysis and interpretation of the data: JP, PB, PJEB, and BMR. Drafting of the manuscript: BMR. Critical revision of the manuscript for important intellectual content: all authors. Statistical analysis: MP, GtR, AK, RG and BMR. Study supervision: JP, PB, PJEB.

We thank NIVEL for their permission to use data from the Second Dutch National Survey of General Practice (DNSGP-2).

Funding: The study was supported by an unrestricted grant from "PICASSO for COPD", an initiative of Pfizer, Boehringer Ingelheim and the research institute Caphri (Care and Public Health Research Institute) from the University of Maastricht, The Netherlands (project 004).

The funding source had no role in the design or conduct of the study, collection, management, analysis, or interpretation of the data, or preparation, review or approval of the manuscript.

Competing interests: None.

\section{REFERENCES}

1. Allegra L, Blasi F, de Bernardi B, et al. Antibiotic treatment and baseline severity of disease in acute exacerbations of chronic bronchitis: a re-evaluation of previously published data of a placebo-controlled randomized study. Pulm Pharmacol Ther 2001:14:149-55.

2. Anthonisen NR, Manfreda J, Warren CP, et al. Antibiotic therapy in exacerbations of chronic obstructive pulmonary disease. Ann Intern Med 1987;106:196-204.

3. Saint S, Bent S, Vittinghoff E, et al. Antibiotics in chronic obstructive pulmonary disease exacerbations. A meta-analysis. JAMA 1995;273:957-60.

4. Ram FSF, Rodriguez-Roisin R, Granados-Navarette A, et al. Antibiotics for exacerbations of chronic obstructive pulmonary disease. Cochrane Database Syst Rev 2006;CD004403.

5. Jorgensen AF, Coolidge J, Pedersen PA, et al. Amoxicillin in treatment of acute uncomplicated exacerbations of chronic bronchitis. A double-blind, placebo-controlled multicentre study in general practice. Scand J Prim Health Care 1992;10:7-11.

6. Sachs AP, Koeter GH, Groenier KH, et al. Changes in symptoms, peak expiratory flow, and sputum flora during treatment with antibiotics of exacerbations in patients with chronic obstructive pulmonary disease in general practice. Thorax 1995;50:758-63.

7. Puhan MA, Vollenweider D, Latshang T, et al. Exacerbations of chronic obstructive pulmonary disease: when are antibiotics indicated? A systematic review. Respir Res 2007:8:30

8. American Thoracic Society/and European Respiratory Society Task Force. Standards for the diagnosis and management of patients with COPD. New York: American Thoracic Society; 2004. http://www.thoracic.org/index.cfm (accessed 14 September 2008)

9. National Collaborating Centre for Chronic Conditions. Chronic obstructive pulmonary disease. National clinical guideline on management of chronic obstructive pulmonary disease in adults in primary and secondary care. Thorax 2004;59(Suppl 1):1-232.

10. Global Initiative for Chronic Obstructive Lung Disease. Global Strategy for the Diagnosis, Management, and Prevention of COPD. Summary of GOLD recommendations, with citations from the scientific literature, updated 2007. http:// www.goldcopd.com/Guidelineitem.asp?!1 = 2\&|2 =1 1 fintld $=996$ (accessed 14 September 2008).

11. Bellamy D, Bouchard J, Henrichsen S, et al. International Primary Care Respiratory Group (IPCRG) Guidelines: management of chronic obstructive pulmonary disease (COPD). Prim Care Respir J 2006;15:48-57.

12. Smeele IJM, Van Weel C, Van Schayck CP, et al. Huisarts Wet 2007;50:362-79.

13. Goossens H, Ferech M, Vander Stichele R, et al. Outpatient antibiotic use in Europe and association with resistance: a cross-national database study. Lancet 2005;365:579-87.

14. PHARMO Institute, Utrecht, The Netherlands. http://www.pharmo.nl (accessed 14 September 2008)

15. Herings RM, Bakker A, Stricker BH, et al. Pharmacomorbidity linkage: a feasibility study comparing morbidity in two pharmacy based exposure cohorts. J Epidemiol Community Health 1992;46:136-40.

16. Lau HS, de BA, Beuning KS, et al. Validation of pharmacy records in drug exposure assessment. J Clin Epidemiol 1997;50:619-25.
17. Roede BM, Bindels PJ, Brouwer HJ, et al. Antibiotics and steroids for exacerbations of COPD in primary care: compliance with Dutch guidelines. Br J Gen Pract 2006;56:662-5

18. Seemungal TAR, Donaldson GC, Bhowmik A, et al. Time course and recovery of exacerbations in patients with chronic obstructive pulmonary disease. Am J Respir Crit Care Med 2000;161:1608-13.

19. Kelly PJ, Lim LL-Y. Survival analysis for recurrent event data: an application to childhood infectious diseases. Statist Med 2000;19:13-33.

20. Therneau TM, Grambsch PM. Modeling survival data: extending the Cox model. New York: Springer-Verlag, 2000:xiii +350

21. Niewoehner DE, Lokhnygina $Y$, Rice $K$, et al. Risk indexes for exacerbations and hospitalizations due to COPD. Chest 2007;131:20-8.

22. NIVEL Institute, Utrecht, The Netherlands. http://www.nivel.nl/international/; http://www.nivel.nl/pdf/ns2_ro_h14.pdf (accessed 14 September 2008)

23. WONCA (World Organization of Family Doctors Classification Committee) ICPC-2-R: International Classification of Primary care, revised 2nd Edn. Singapore: WONCA, 2005.

24. Stukel TA, Fisher ES, Wennberg DE, et al. Analysis of observational studies in the presence of treatment selection bias: effects of invasive cardiac management on AMI survival using propensity score and instrumental variable methods. JAMA 2007:17:297:278-85.

25. Sethi $\mathbf{S}$. The role of antibiotics in acute exacerbations of chronic obstructive pulmonary disease. Curr Infect Dis Rep 2003:5:9-15.

26. Hoogendoorn M, Feenstra TL, Schermer TR, et al. Severity distribution of chronic obstructive pulmonary disease (COPD) in Dutch general practice. Respir Med 2006;100:83-6.

27. El Moussaoui R, Roede BM, Speelman P, et al. Short course antibiotic treatment in acute exacerbations of COPD: a meta-analysis of double-blind studies. Thorax 2008:63:415-22.

28. Murphy TF, Sethi $S$, Niederman MS. The role of bacteria in exacerbations of COPD. A constructive view. Chest 2000;118:204-9.

29. Perera WR, Hurst JR, Wilkinson TMA, et al. Inflammatory changes, recovery and recurrence at COPD exacerbation. Eur Respir J 2007;29:527-34.

30. Bresser $\mathbf{P}$, Out $\mathrm{TA}$, van $\mathrm{AL}$, et al. Airway inflammation in nonobstructive and obstructive chronic bronchitis with chronic haemophilus influenzae airway infection Comparison with noninfected patients with chronic obstructive pulmonary disease. Am J Respir Crit Care Med 2000;162:947-52.

31. Bresser $\mathbf{P}$, van AL, Habets FJ, et al. Persisting Haemophilus influenzae strains induce lower levels of interleukin-6 and interleukin-8 in H292 lung epithelial cells than nonpersisting strains. Eur Respir J 1997:10:2319-26.

32. Ketterer MR, Shao JQ, Hornick DB, et al. Infection of primary human bronchial epithelial cells by Haemophilus influenzae: macropinocytosis as a mechanism of airway epithelial cell entry. Infect Immun 1999;67:4161-70.

33. Moller LV, Timens W, van der Bij W, et al. Haemophilus influenzae in lung explants of patients with end-stage pulmonary disease. Am J Respir Crit Care Med 1998;157:950-6.

34. Sethi S, Evans N, Grant BJ, et al. New strains of bacteria and exacerbations of chronic obstructive pulmonary disease. N Engl J Med 2002;15:347:465-71.

35. Stockley RA, O'Brien C, Pye A, et al. Relationship of sputum color to nature and outpatient management of acute exacerbations of COPD. Chest 2000;117:1638-45.

36. Gompertz S, O'Brien C, Bayley DL, et al. Changes in bronchial inflammation during acute exacerbations of chronic bronchitis. Eur Respir J 2001;17:1112-19.

37. Bronzwaer SL, Cars 0, Buchholz U, et al. A European study on the relationship between antimicrobial use and antimicrobial resistance. Emerg Infect Dis 2002;8:278-82.

38. Finch RG. Introduction: standards of antibacterial performance. Clin Microbiol Infect 2004;10(Suppl 2):1-5.

39. Hsueh PR, Shyr JM, Wu JJ. Changes in macrolide resistance among respiratory pathogens after decreased erythromycin consumption in Taiwan. Clin Microbiol Infect 2006; 12:296-8

40. Chen DK, McGeer A, de Azavedo JC, et al. Decreased susceptibility of Streptococcus pneumoniae to fluoroquinolones in Canada. Canadian Bacterial Surveillance Network. N Engl J Med 1999;341:233-9.

41. Felmingham D, Gruneberg RN. The Alexander Project 1996-1997: latest susceptibility data from this international study of bacterial pathogens from community-acquired lower respiratory tract infections. J Antimicrob Chemother 2000;45:191-203. 